\title{
Measurement Stand for TeraEYE Inspection
}

\author{
M. PiszczeK*, M. Kowalski And M. Szustakowski \\ Institute of Optoelectronic, Military University of Technology, S. Kaliskiego 2, 00-908 Warsaw, Poland \\ Image fusion can be used as one of imaging methods. Connection of images acquired using different \\ acquisition methods can deliver information invisible for traditional one imaging technique. Visible and thermal \\ images connected in one image give information about shapes and temperature map of objects seen by cameras. \\ If we connect stereoscopic visible image and thermal image we will have three-dimensional object model covered \\ with temperature map. Dividing three-dimensional objects on elementary surfaces we can model this object \\ energetically. Energetic modeling of measurement scene allows verifying measurement data acquired from devices \\ working in terahertz frequency range. This method requires many measurement scene parameters to be registered. \\ Some of these parameters can be used in a hybrid modelling process.
}

PACS: 02.60.-x, 42.30.Tz, 42.30.Sy

\section{Introduction}

The Institute of Optoelectronics at the MUT is taking part in the European TeraEYE Project. The purpose of this project is to develop a cheap, innovative passive terahertz security system to detect dangerous and harmful materials. Such a system could be applied to airport security systems as well as crowded and public places with security systems e.g. train stations, subway stations etc. [1-6]. This system could be also used to detect dangerous chemicals and organic substances hidden in deliveries.

The aim of the TeraEYE project is to revolutionize terahertz technology by developing a completely new nanoscale production process for cheap, two-dimensional matrices of detectors e.g. antennas, quantum dots, single electron transistors (SETs). Development of THz detectors could reduce the high costs of $\mathrm{THz}$ sensors production.

Within the framework of the TeraEYE project:

— works on detector were performed (Fig. 1).

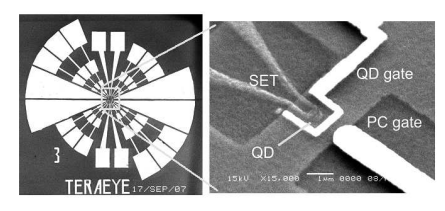

Two types of sensor were tested: QD-SET and QD-PC

\begin{tabular}{|c|c|c|}
\hline & SETQD & PGQQD \\
\hline $\begin{array}{l}\text { Control } \\
\text { electrodes }\end{array}$ & $2+1$ & $3+1$ \\
\hline $\begin{array}{l}\text { Working } \\
\text { temperature }\end{array}$ & $0.3-0.7 \mathrm{~K}$ & Upt t $1.5 \mathrm{~K}$ \\
\hline Operation & $5 \mathrm{mV}$ & $50 \mathrm{mv}$ \\
\hline NEP & $10^{17} \mathrm{~W}_{1 / 1 \mathrm{z}^{1 / 2}}$ & $10^{-15} \mathrm{WHHz}^{12}$ \\
\hline Time resolution & $0.1 \mathrm{~ms}$ & $0.01 \mathrm{~ms}$ \\
\hline
\end{tabular}

Fig. 1. TeraEYE detector and parameters.

- there were worked out: antennas matrix configuration, cooling system and optical windows (Fig. 2),

- electronic circuits of device were worked out (Fig. 3),

- preliminary device tests and compact housing development at the moment.

* corresponding author; e-mail: mpiszczek@wat.edu.pl

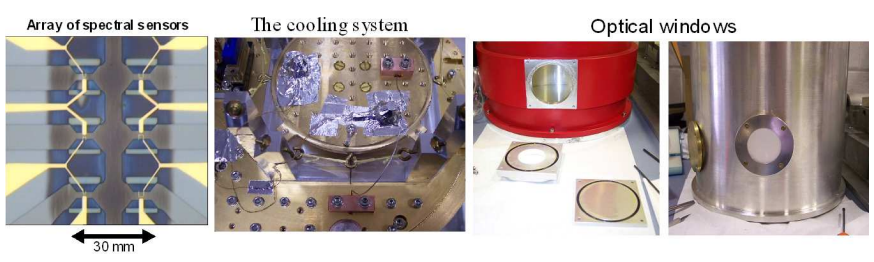

Fig. 2. TeraEYE components.

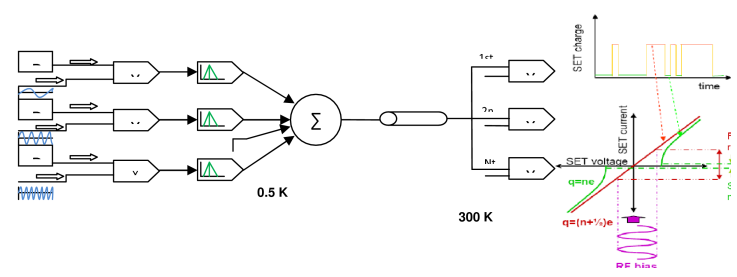

Fig. 3. TeraEYE electronics.

One of the tasks of the Polish part of the TeraEYE project is testing the TeraEYE device in real conditions (Fig. 4). To accomplish this task it was necessary to prepare a measurement station as well as organize a measurement process. Future measurements will be made in real conditions because there is a need to register the parameters of every measurement session as well as data acquisition. Measurement parameters are required to
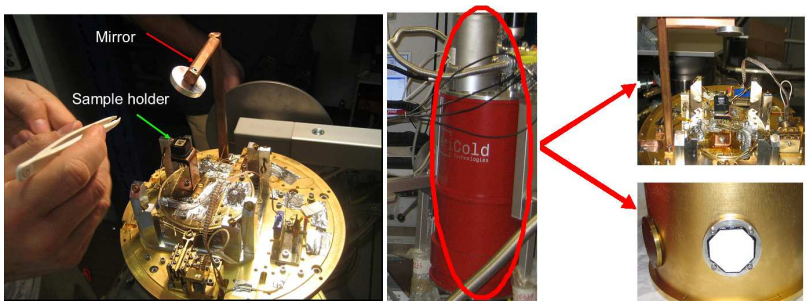

Fig. 4. Prototype of TeraEYE device. 


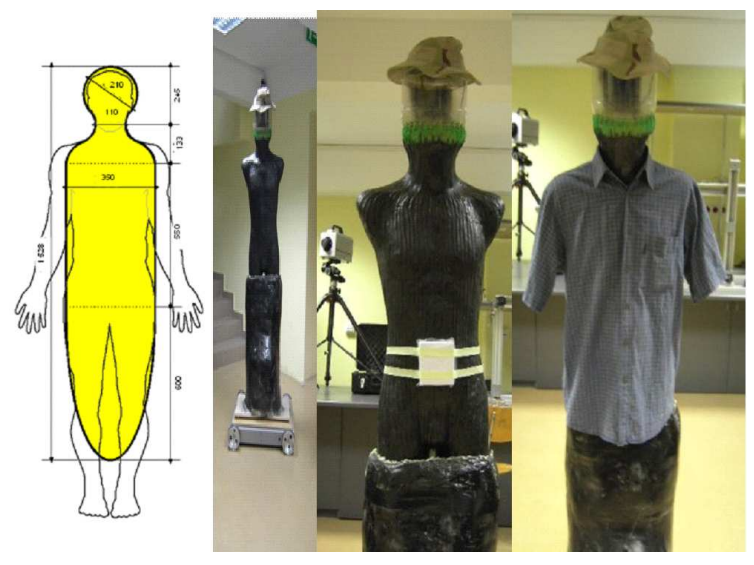

Fig. 5. Thermal phantom.

validate the data acquired and for the eventual correction method. One of the issues regarded in TeraEYE device testing is to model the measurement scene. Ability of correct measurement data interpretation comes from capability of proper $\mathrm{THz}$ sources modelling. For automatic data acquisition there is a vision system equipped with cameras working in the visible and infrared spectral range. For three-dimensional visualization and hybrid modelling we built a thermal phantom, which is a model of a human shape (Fig. 5).

\section{TeraEYE measurement characteristics}

One of the most important aspects of real conditions of TeraEYE device testing is to suggest a proper method of data validation. Research on passive $\mathrm{THz}$ detection is in its infancy so there is no other measurement method we can use to validate data. Our proposed solution is based on energetic parameters modelling in the $\mathrm{THz}$ frequency range. This method requires many measurement scene parameters to be registered. Some of these parameters can be used in a hybrid modelling process. Besides acquisition of local climatic parameters (e.g. temperature, air pressure, humidity) registration of the measurement object parameters plays a key role [7]. During the testing process we will use dangerous materials e.g. explosives and for this reason we have built a thermal phantom (a dummy made of non-metallic materials, which is important for research in the $\mathrm{THz}$ range) for better shape and energetic modeling [2] (Fig. 5).

$\mathrm{THz}$ modelling using the thermal phantom needs geometric and energetic modelling of the measurement object for every measurement session. Every session will consist of measurements taken in many different locations and orientations of the measurement object as well as with various dangerous materials. To achieve the design demands and post-process acquired data there is a need to build photogrametric frame. The role of the frame is to acquire and process data using an image fusion algorithm.

\section{Measurement process arrangement - hybrid model}

$\mathrm{THz}$ energetic modelling requires the parameters of the elementary radiating surfaces to be known. For the thermal phantom we consider surface geometry and its orientation in space with information about temperature (Fig. 6).

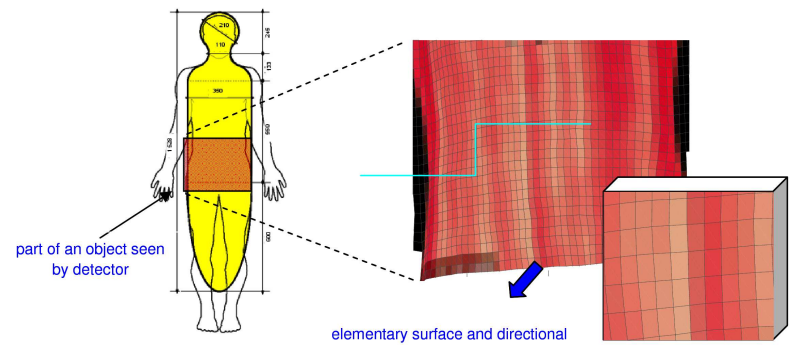

Fig. 6. Elementary surface of thermal phantom model.

The energetic modeling procedure is concerned with estimating the power reaching the $\mathrm{THz}$ detector in its sensitivity range. Among calculations we take into account:

a) power emitted by the black body elementary surface to half-sphere. It can be calculated using Planck's law

$$
P_{\mathrm{b}}=\int_{\lambda_{1}}^{\lambda_{2}} \frac{\pi h c^{2}}{\lambda^{5}\left(\mathrm{e}^{\frac{h c}{\lambda k T}}-1\right)} \mathrm{d} \lambda ;
$$

b) emissivity $\varepsilon$ of the radiating object, because such an object is not a black body. The power emitted by an elementary surface can be calculated by the equation below

$$
P_{\mathrm{t}}=P_{\mathrm{b}} A \varepsilon
$$

c) geometrical parameters of the measurement path such as the lens surface $S$ and its distance from the object $R$ making it possible to calculate the solid angle $\Omega$. This is the angle between elementary surface and detection system. Parameter $\Omega$ tells us how much of the emitted radiation reaches the lens

$$
\Omega=\frac{S}{R^{2}} .
$$

Additionally, if we know $\alpha$ angle between the emitting surface and the rays direction, we can consider cosine distribution of radiation

$$
P=P_{x} \cos \alpha ;
$$

d) air transmission characteristics $T$ (in constant temperature, humidity, wavelength and distance) and optics attenuation $D$ allows us to calculate the total power reaching the detector

$$
P=P_{\mathrm{t}} T D \frac{\Omega}{2 \pi} \cos \alpha .
$$

The procedure described above shows how important hybrid modelling is for calculating power emitted by the elementary surface. 
From a point of view of measurement session there is no need to build a complete 3D model but just a part of the object's surface seen by the THz detector (Fig. 6).

To acquire all the necessary data for three-dimensional model synthesis we used a photogrametric frame equipped with VIS and IR cameras [7]. In the hybrid modelling technique images acquired using different sources are combined into one image [8]. As a result of data fusion many parameters impossible to extract using standard techniques of visualization can be seen. This synergy effect which comes along in hybrid modelling gives all the necessary information for $\mathrm{THz}$ modelling.

Due to measurements can be realized in e.g. closed area (electromagnetic tent) with different types of covering materials, structural light can be used to improve data post-processing methods (Fig. 7).

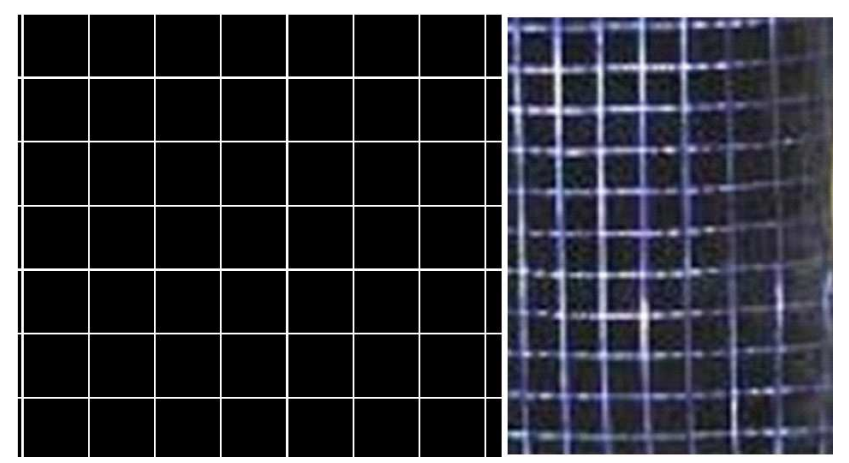

Fig. 7. Snapshot of electronically generated net and part of structurally lit phantom surface.

Hybrid modelling procedure (which is part of the measurement process, Fig. 8) needs:

- measurement frame calibration (before the actual measurement process),

- acquisition of VIS and IR images of structurally lit thermal phantom,

- spatial model synthesis,

— hybrid model synthesis.

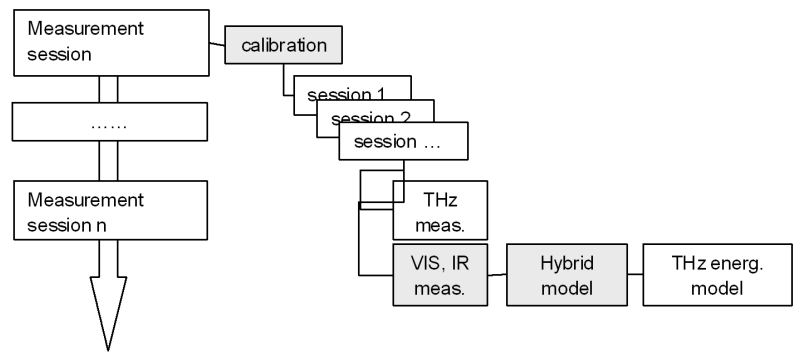

Fig. 8. Analytic - measurement process arrangement for TeraEYE device.

\subsection{Calibration}

A calibration procedure has to be carried out before every measurement session. This is necessary because the measurement area is different in every measurement session and has to be defined. For proper calibration a test object (control) is used. The test object is a matrix of 49 markers $7 \times 7$ matrix) for VIS cameras and 4 markers for IR cameras (Fig. 9).

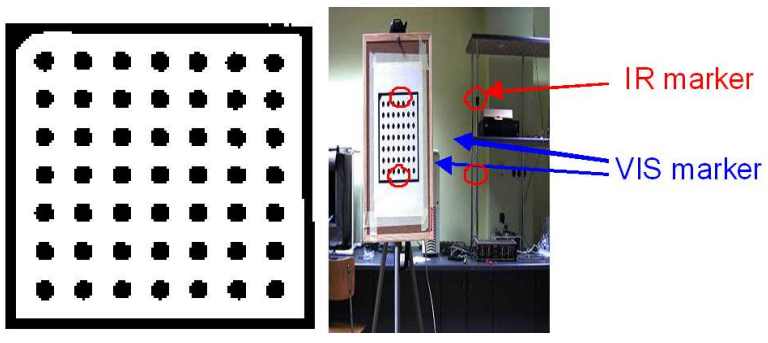

Fig. 9. Calibration test object.

Using the test object presented above gives us the possibility to work out a calibration procedure in which stereo-pair VIS images and corresponding IR thermal images in different spatial locations of the test object are acquired. Use of a personal computer as a control unit makes the calibration process automated. Automation of calibration process regards test objects space location steering and data verification algorithms. The calibration parameters (elements of internal and external orientation of the VIS cameras) are calculated based on verified test data and are used for triangulation and projection transition for connecting spatial coordinates with the thermal image.

\subsection{Modeling}

Calibration coefficients can be used for measurement data analysis. During every measurement session image triplets (VIS1, VIS2 and IR) of the thermal phantom are acquired. An automated structural light and proper image analysis algorithms procedure for finding homologous points for images VIS1 and VIS2 is used. Spatial coordinates of homologous points computed in the triangulation procedure make a 3D measurement object model possible. Spatial data and thermal image fusion make possible hybrid modelling and a 3D thermal model of the thermal phantom. Climatic parameters of measurement space and 3D modelling results are data required for energetic modelling in the $\mathrm{THz}$ frequency range.

\section{Measurement frame}

Taking measurements with proper spatial and temperature resolution is connected with the necessity of using precise measurement tools. The possibility of proper space mapping and measurement conditions was one of the main problems when considering the selection of components for the photogrametric frame. 
For the TeraEYE project a special photogrametric frame was built for image acquisition and spatial measurement parameters modelling. This frame consisted of two IQinVision IQeve 755 cameras working in the visible spectral range, FLIR A320 thermal camera and BENQ W6744 projector (as structural illuminator) (Fig. 10).
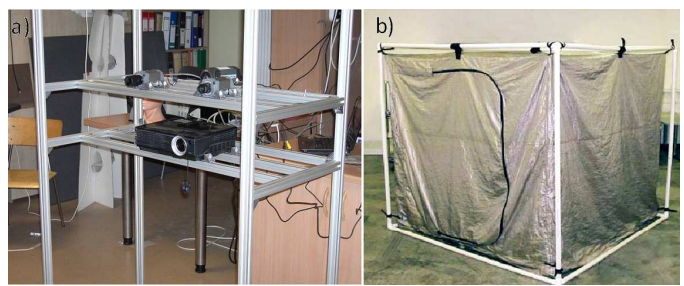

Fig. 10. Measurement frame (a) photogrametric frame (b) electromagnetic tent.

VIS cameras were equipped with Computer MEGAPIXEL lenses with adjustable focal length.

All cameras were connected to a computer unit using Ethernet line. To collect data from cameras data acquisition cards (Intel and RealTEK) were used. Ethernet lines were used for bi-directional communication with cameras - acquiring data and sending control commands.

One of the biggest problems encountered during building the measurement position was process automation. Cameras were controlled using specially prepared software. The control program was developed in an object programming language using Microsoft Visual Studio 2008. The control software developed can be used to change cameras measurement parameters e.g. change the type of structural illumination, and automatically save acquired data.

\section{Hybrid modeling results}

The calibration methods and measurement frame were tested with real measurement data. Three cameras placed on the frame were controlled by a central PC equipped with Windows. During the first measurement session a few image triplets were acquired of the test object placed on the control platform.
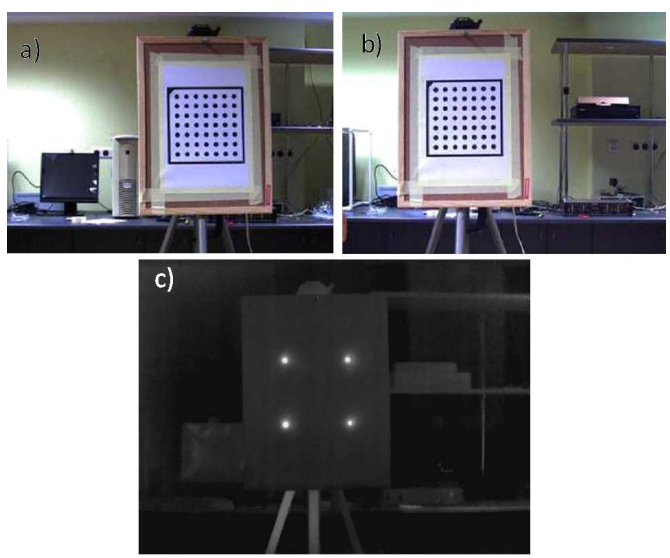

Fig. 11. Test object (a) left VIS, (b) right VIS, (c) thermal.

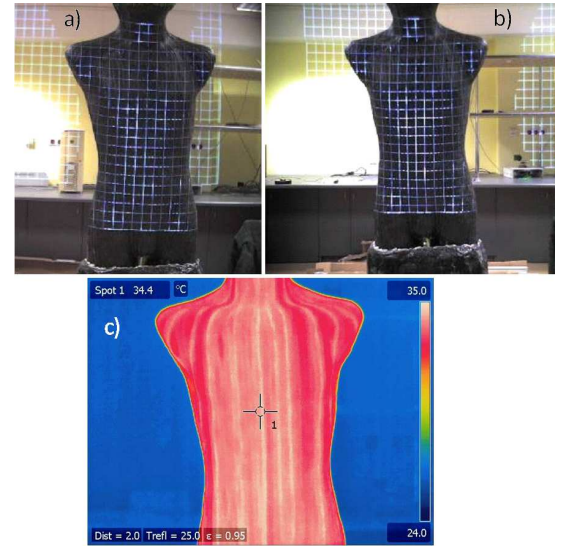

Fig. 12. Set of measurement images (a) left VIS, (b) right VIS, (c) thermal.

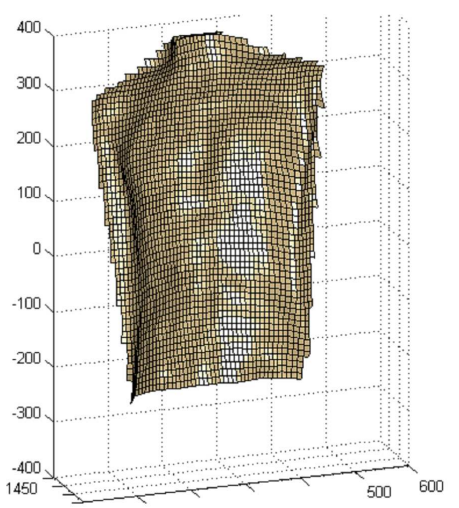

Fig. 13. Three-dimensional phantom model.

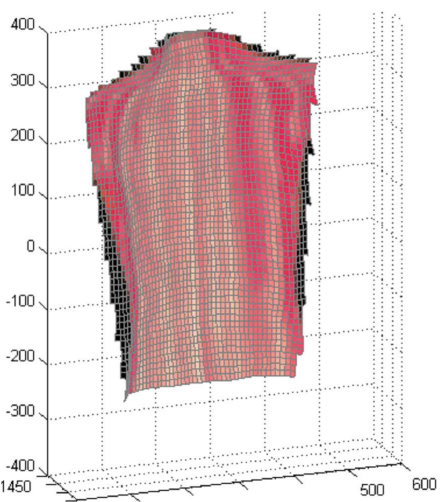

Fig. 14. Hybrid model of thermal phantom.

Tests were based on a series of images with calibrated VIS and IR cameras (Fig. 11). The calibration gave us a set of parameters for use in hybrid modeling [8].

Proper measurement process relies on image acquisition of structurally lit thermal phantom. Acquired images are presented in Fig. 12. 
Measurement data analysis using calibration data and the triangulation procedure enables assignment of homologous points on the phantom's surface to 3D spatial points. A 3D model of the thermal phantom is a result of this operation (Fig. 13).

Data post-processing algorithms were used to synthesize a hybrid model based on data from the thermal camera and 3D phantom model. As a result of data fusion there is a hybrid model of thermal phantom presented in Fig. 14.

The calibrated photogrametric frame can be used to model measurement conditions. Estimating parameters of space is one of the most important factors with testing the TeraEYE device.

\section{Summary}

Development of $\mathrm{THz}$ devices technology necessitates being able to verify received measurement data unambiguously. At present $\mathrm{THz}$ technology development does not let to verify correctness of received data properly. This is the purpose of inventing new research methods which can be useful as reference methods. In the case of research on $\mathrm{THz}$ devices lead in Institute of Optoelectronics, MUT we used photogrametric measurement position equipped with VIS and IR cameras.

Data fusion can be used to visualize a few different types of information in the same image. Our invented data fusion method which combines stereoscopic VIS images and a IR thermal image allows us to show a temperature map on a three-dimensional object. This method was used in $\mathrm{THz}$ radiation research. The ability to ver- ify received data depends on the ability to model $\mathrm{THz}$ sources and elements of the measurement scene. Three-dimensional objects with map of temperature can be used to model human's shape.

Proposed photogrammetric position was used in $\mathrm{THz}$ research. However, this is not the only application of this position because hardware configuration as well as the data acquisition method are universal and can be used in any research.

\section{References}

[1] R. Ryniec, M. Piszczek, M. Szustakowski, Acta Phys. Pol. A 118, 1235 (2010).

[2] N. Pałka, Acta Phys. Pol. A 118, 1229 (2010).

[3] H. Quast, T. Löffler, 3D-Terahertz-Tomography for Material Inspection and Security, SynView GmbH, Glashuetten, Germany 2009.

[4] J.A. Walkenstein, W.B. Pardo, H.S. Robertson, M. Monti, Rev. Sci. Instrum. 11, 5385 (1995).

[5] T. Pustelny, C. Tyszkiewicz, K. Barczak, Opt. Appl. 33, 469 (2003).

[6] E. Maciak, Z. Opilski, T. Pustelny, M. Bednorz, J. Phys. IV (France) 129, 131 (2005).

[7] X. Su-xia, G. Pei-yuan, C. Tian-hua, in: Proc. Int. Conf. on Measuring Technology and Mechatronics Automation, Paris (France) 2010, ICAL, Paris 2010, p. 347.

[8] Q. Wang, Yi. Shen, in: IMTC 2004 - Proc. Instrumentation and Measurement Technology Conf., IMTC, Coma 2004, p. 265. 